\title{
Ensemble Effect Evidenced by CO Adsorption on the 3-Fold PdGa Surfaces
}

\author{
Jan Prinz, ${ }^{\dagger, \ddagger}$ Roberto Gaspari, ${ }^{\dagger, \S}$ Quirin S. Stöckl, ${ }^{\|}$Peter Gille, ${ }^{\perp}$ Marc Armbrüster, ${ }^{\#}$ Harald Brune, ${ }^{\ddagger}$ \\ Oliver Gröning, ${ }^{\dagger}$ Carlo A. Pignedoli, ${ }^{\dagger}$ Daniele Passerone, ${ }^{\dagger}$ and Roland Widmer*, ${ }^{\dagger}$
}

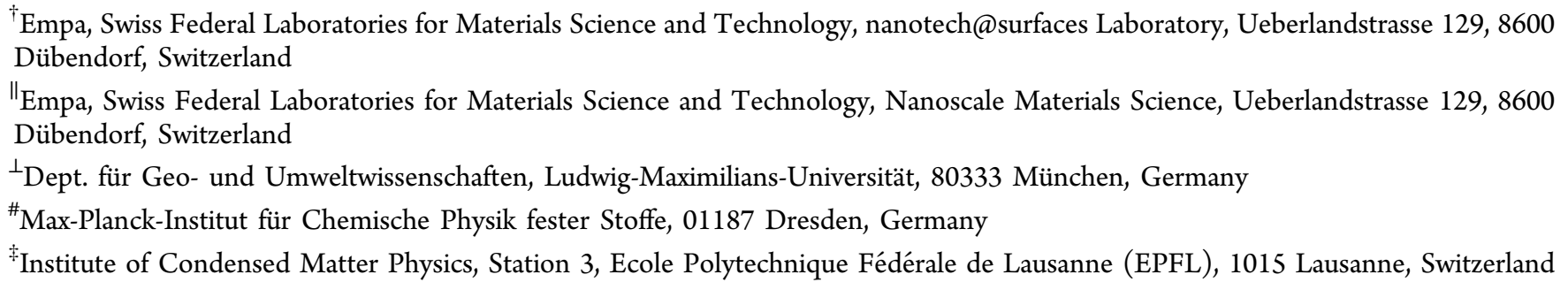

Supporting Information

ABSTRACT: The atomic structure and composition of a catalyst's surface have a major influence on its performance regarding activity and selectivity. In this respect, intermetallic compounds are promising future catalyst materials, as their surfaces exhibit small and well-defined ensembles of active metal atoms. In this study, the active adsorption sites of the 3-fold-symmetric surfaces of the PdGa intermetallic compound were investigated in a combined experimental and computational approach using $\mathrm{CO}$ as a test molecule. The $\mathrm{PdGa}(111)$ and $(-1-$ $1-1)$ surfaces exhibit very similar electronic structures, but have Pd sites with very different, well-defined atomic coordination and separation. They thereby serve as prototypical model systems for studying ensemble effects on bimetallic catalytic surfaces. Scanning tunneling microscopy and Fourier transform infrared spectroscopy show that the $\mathrm{CO}$ adsorption on both surfaces is solely associated with the topmost Pd atoms and Ga acts only as an inactive spacer. The different local configurations of these $\mathrm{Pd}$ atoms dictate the $\mathrm{CO}$ adsorption sites as a function of coverage. The experimental results are corroborated by density functional theory and illustrate the site separation and ensemble effects for molecular adsorption on intermetallic single crystalline surfaces.

\section{INTRODUCTION}

Surface science methods allow for studying the structure of catalytic surfaces on the atomic level, and in some cases even under reaction pressures. ${ }^{1,2}$ This ability expanded the understanding of heterogeneous catalysis and led to many ideas on how to structure catalyst surfaces to improve their performance. $^{3-6}$ An important aspect of research in catalysis, besides achieving high activity, is improving selectivity. One proposed enhancement is the dilution of the active (metal) species with less active spacer atoms. This site isolation concept consists of forming isolated catalytic centers of reduced size, leading to a limited number of adsorption configurations for the reactants, which is called the "ensemble effect". ${ }^{7-9}$ The number of reaction pathways emerging from these active centers is thereby reduced, resulting in an increased selectivity, as compared to metal surfaces consisting of a single element.

In addition to this ensemble effect, the electronic structure of the active species is influenced by the nature and concentration of the spacer atoms, which is known as the "ligand effect".,9 Since atomic and electronic structure of a surface are always interconnected, it is difficult to unambiguously impute a given experimental observation purely to the ligand-, and/or to the ensemble effect of a catalytic surface.

One model system in this respect is bimetallic surface alloys, which have a random spatial distribution of active sites. $3,7-11$ Intermetallic compounds (IMCs) are a second model system, which have an ordered crystal structure. Additionally, they exhibit a higher energy of formation, which potentially increases surface stability., ${ }^{3,-12}$ In this respect, the PdGa IMC has recently received considerable interest due to its high activity, stability, and selectivity in the semihydrogenation of acetylene. ${ }^{12,13}$ This reaction is industrially important, ${ }^{14}$ as it is a crucial step in polyethylene production, where currently $\mathrm{Ag}-$ Pd alloy catalysts are applied. In the context of the site isolation concept, also for PdGa, Pd is supposed to take the role of the catalytically active species and $\mathrm{Ga}$ the role of the spacer atom.

In a recent publication, ${ }^{15}$ we reported on the 3 -fold surfaces of the PdGa IMC. The two crystallographically opposed

Received: February 13, 2014

Revised: $\quad$ May 14, 2014

Published: May 14, 2014 
surfaces, (111) and (-1-1-1), exhibit equal surface symmetry, equal lattice parameters, and very similar electronic structure, but different atomic configurations of the Pd atoms. On one surface separated Pd trimers are present, while the other is terminated by isolated, single $\mathrm{Pd}$ atoms. This particular situation allows for investigating the pure structural ensemble effect, while the influences from the ligand effect can be neglected.

On the other hand, studying the ligand effect can be accomplished by exchanging the chemical species of the spacer atoms, while keeping the atomic surface structure unchanged. Whereas the realization of this concept is experimentally challenging, it has been used in theoretical studies, such as in quantum chemical calculations performed by Nørskov et al. ${ }^{16}$ This computational screening for improved catalysts reveals trends for the selection of the most promising constituent materials.

In this study, we present experimental and computational results of $\mathrm{CO}$ adsorption on the $\mathrm{PdGa}: \mathrm{B}(-1-1-1) \mathrm{Pd}_{1}$ and PdGa:B(111) $\mathrm{Pd}_{3}$ surfaces. ${ }^{15}$ These two surfaces serve as prototypical system to study the ensemble effect because they expose two different terminations. One consisting of single palladium atoms $\left(\mathrm{Pd}_{1}\right)$ and the second of palladium trimers $\left(\mathrm{Pd}_{3}\right)$. The effects of the Pd site structure are directly observed in the vibrational frequency of the adsorbed $\mathrm{CO}$ as a function of coverage as measured by Fourier transform infrared spectroscopy (FTIR). Scanning tunneling microscopy (STM) provides local information on the adsorption geometry and allows an unambiguous interpretation of the vibrational spectroscopy. Density functional theory (DFT) calculations agree with the experimental results and allow for an in depth understanding of the bonding situation of $\mathrm{CO}$ to the single atomic and trimer Pd sites.

We observe strong site isolation effects on both PdGa surfaces, which are most clearly expressed on the PdGa:B(-1$1-1) \mathrm{Pd}_{1}$ surface, where the full $\mathrm{CO}$ coverage at lowtemperature $(77 \mathrm{~K})$ and pressure $\left(10^{-6}\right.$ mbar range $)$ is about $15 \%$ of that of $\operatorname{Pd}(111)$. To the best of our knowledge, this is the first experimental demonstration of the ensemble effect on well-defined intermetallic compound surfaces, which is a promising class of materials for the development of future catalysts.

\section{METHODS}

Experiments. The growth and surface preparation of the PdGa single crystals has been described in detail previously, so that only a brief summary is given. ${ }^{17,18}$ The PdGa single crystal surfaces were cleaned by Ar-ion sputtering and high temperature annealing $(870 \mathrm{~K})$ cycles. $^{15,19}$ The STM measurements were performed with an Omicron low-temperature (LT-) STM at a base pressure below $5 \times 10^{-11}$ mbar using a mechanically cut Pt/Ir-tip.

FTIR absorption spectroscopy measurements were recorded with a Bruker Vertex $70 \mathrm{v} / 80 \mathrm{v}$ spectrometer in reflectionabsorption mode (RAIRS). CO (purity 4.7, CANGas) was dosed on the sample at $90 \mathrm{~K}( \pm 10 \mathrm{~K})$ by backfilling the main chamber.

Simulations. All simulations were performed within DFT in the mixed Gaussian Plane Waves framework as implemented in the $\mathrm{CP} 2 \mathrm{~K}$ code (see SI for further details). ${ }^{20}$ We used the Perdew-Burke-Ernzerhof $(\mathrm{PBE})^{21}$ parametrization for the exchange correlation functional.
PdGa slabs were simulated in the repeated slab geometry. ${ }^{22}$ Orthorhombic simulation cells of 23 and 24 atomic PdGa layers were used for simulations of the PdGa:B(111) and PdGa:B(-1-1-1) surface, respectively. The positions of the lowest eight layers were fixed in the structure optimization. About $3 \mathrm{~nm}$ of vacuum was added above the adsorbate atoms. The lateral dimension of the cell corresponds to 12 hexagonal surface unit cells. The ground state adsorption energies and structures were computed by relaxing all atomic positions until forces were lower than $0.05 \mathrm{eV} / \mathrm{nm}$. IR spectra were calculated using finite differences starting from the equilibrium adsorption geometries. $^{20}$

\section{RESULTS AND DISCUSSION}

A first approach to determine the adsorption sites of $\mathrm{CO}$ on $\mathrm{PdGa}: \mathrm{B}(111) \mathrm{Pd}_{3}$ and $(-1-1-1) \mathrm{Pd}_{1}$ was performed by LTSTM measurements. Figure 1 shows STM images of both

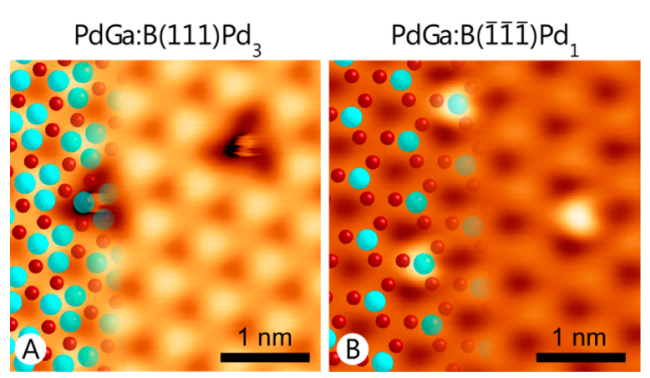

Figure 1. STM images of $\mathrm{CO}$ adsorbed on $\mathrm{Pd}_{3}(\mathrm{~A})$ and $\mathrm{Pd}_{1}(\mathrm{~B})$. The atomic surface structure is overlaid on the left-hand site of each image (Pd: cyan, large; Ga: red, small). The top layer consists of Pd trimers, for $\mathrm{Pd}_{3}(\mathrm{~A})$, and single $\mathrm{Pd}$ atoms for $\mathrm{Pd}_{1}(\mathrm{~B})$. The second layer consists of $\mathrm{Ga}$ trimers for both surface orientations. On $\mathrm{Pd}_{3}(\mathrm{~A})$, two $\mathrm{CO}$ molecules are present, appearing as triangular depressions. On $\mathrm{Pd}_{1}$ (B), three $\mathrm{CO}$ molecules are present, appearing as protrusions (Tunneling parameters: A: $-7 \mathrm{mV}, 2 \mathrm{nA}$; B: $-1 \mathrm{~V}, 35 \mathrm{nA}$ ).

surfaces at very low $\mathrm{CO}$ coverage. The surface $\mathrm{Pd}$ atoms are imaged as protrusions. ${ }^{15}$ In combination with low energy electron diffraction (LEED) measurements and STM simulations, the atomic structure of the clean surface is identified with regard to lateral position and orientation, ${ }^{15}$ and overlaid on the left-hand side of each image for the two surfaces. The samples were exposed to $\mathrm{CO}$ during imaging at $78 \mathrm{~K}$, which allowed for identifying single adsorbed $\mathrm{CO}$ molecules. On $\mathrm{Pd}_{3}$, $\mathrm{CO}$ molecules appear as triangular depressions with a central maximum, whereas on $\mathrm{Pd}_{1}$, they are imaged as bright protrusions. According to the atomic structure overlay, individual $\mathrm{CO}$ molecules adsorb in the 3-fold Pd hollow site on $\mathrm{Pd}_{3}$, and at the on-top Pd site on $\mathrm{Pd}_{1}$.

To complement these atomic scale observations and to study the adsorption kinetics, RAIRS experiments as a function of $\mathrm{CO}$ coverage were carried out. As shown in Figure 2, a single, $\mathrm{CO}$ coverage independent, absorption peak is observed for the $\mathrm{Pd}_{1}$ surface. For the $\mathrm{Pd}_{3}$ surface, two separate, coverage dependent peaks are observed. Comparison of the peak positions with RAIRS of $\mathrm{CO}$ on $\mathrm{Pd}(111)$ allows for an assignment of the peaks at higher and lower wavenumbers found for $\mathrm{CO} / \mathrm{Pd}_{3}$ to on-top and hollow site adsorption, respectively. ${ }^{3,23}$ This assignment is substantiated by the sequence of binding energies of the optimized adsorption configurations found by DFT for adsorption of one, two, and three CO per Pd trimer (see Table 1 and Supporting 


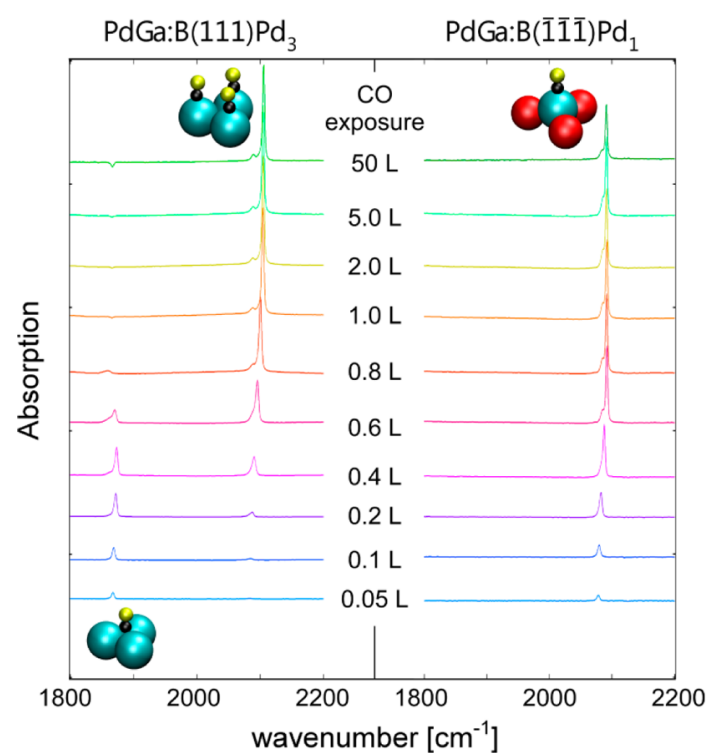

Figure 2. IR absorption spectra as a function of wavenumber and $\mathrm{CO}$ exposure at $90 \mathrm{~K}$ on $\mathrm{Pd}_{3}$ (left) and $\mathrm{Pd}_{1}$ (right). Insets next to the peaks show the respective adsorption configuration ( $\mathrm{C}$ in black, $\mathrm{O}$ in yellow).

Information). Therefore, the series of RAIRS spectra for the $\mathrm{CO} / \mathrm{Pd}_{3}$ can be interpreted as a transition from hollow to ontop site with increasing exposure. ${ }^{3,23}$ A qualitative comparison to similar experiments of $\mathrm{CO} / \mathrm{Pd}(111)$ reveals that peak shapes are sharper and coverage-dependent peak shifts are much smaller for the $\mathrm{CO} / \mathrm{PdG}$ system presented here. Indeed, on

Table 1. Experimental and Theoretical IR Absorption Peak Positions for CO on the Different Sites of the PdGa Surfaces and Calculated Binding Energies ${ }^{a}$

\begin{tabular}{llll} 
& \multicolumn{1}{c}{$\begin{array}{c}\mathrm{CO} / \mathrm{Pd}_{3} \\
\text { (hollow site) }\end{array}$} & \multicolumn{1}{c}{$\begin{array}{c}\mathrm{CO} / \mathrm{Pd}_{3} \\
\text { (on-top site) }\end{array}$} & $\begin{array}{c}\mathrm{CO} / \mathrm{Pd}_{1} \\
\text { (on-top site) }\end{array}$ \\
$\nu_{\text {exp }}\left(\mathrm{cm}^{-1}\right)$ & $1868-1874$ & $2084-2105$ & $2078-2092$ \\
$\nu_{\text {th }}\left(\mathrm{cm}^{-1}\right)$ & 1822 & $2039(2 \mathrm{CO})$ & 2032 \\
& & $2047(3 \mathrm{CO})$ & \\
$E_{\mathrm{b}} / \mathrm{CO}(\mathrm{eV})$ & 1.59 & $1.28(2 \mathrm{CO})$ & 1.37 \\
& $1.25(3 \mathrm{CO})$ & \\
$\begin{array}{c}\mathrm{Pd}-\mathrm{C} \text { bond } \\
\text { order }\end{array}$ & $1.34(3 \times 0.45)$ & 0.82 & 0.83 \\
$\begin{array}{c}\mathrm{C}-\mathrm{O} \text { bond } \\
\text { order }\end{array}$ & 0.91 & 0.98 & 0.95 \\
\hline
\end{tabular}

${ }^{a}$ The covalent bond orders are computed according to Angyan. ${ }^{29}$ For a $\mathrm{CO}$ molecule in the gas phase the theory predicts a value of 1.51 , accounting for the polar character of the $\mathrm{C}-\mathrm{O}$ bond $(2.89$ for the covalent triple bond in $\mathrm{C}_{2} \mathrm{H}_{2}$ ).

$\mathrm{CO} / \mathrm{Pd}(111)$, a full hierarchy of bonding sites is observed (hollow, bridge, and on-top sites), with a coverage-dependent spectrum influenced by long-ranged intermolecular interactions, ${ }^{23-25}$ leading to broadened IR-absorption features. Accordingly, our RAIRS data clearly indicates a suppression of CO-CO interactions on both PdGa surfaces. This is most pronounced in the $\mathrm{CO} / \mathrm{Pd}_{1}$ case, where the on-top sites are separated by $0.69 \mathrm{~nm}$, but is also seen for $\mathrm{CO} / \mathrm{Pd}_{3}$, where at full coverage, the interaction is mainly reduced to the three neighboring $\mathrm{CO}$ molecules on the same trimer. Additionally, the Pd-Pd nearest neighbor distance of $0.3 \mathrm{~nm}$ within such a $\mathrm{Pd}$ trimer is $10 \%$ larger than in $\operatorname{Pd}(111) .^{12}$
A summary of the experimental IR vibration frequencies is shown in Table 1, together with simulated frequencies from DFT for the most stable adsorption geometries, which corroborate the experimental vibrational assignments. The ranges given for the experimental frequencies represent the coverage-dependent variations in peak positions. The computed vibration frequencies underestimate the experimental values by a constant scaling of $2.80 \pm 0.02 \%$. This is a common problem in DFT with PBE approximation (see refs 11 and 26) and can vary according to the parametrization employed. ${ }^{27}$ However, the agreement of the relative frequencies by less than $0.5 \%$ confirms the hypothetical structure model used in DFT.

The combined experimental and theoretical data enables us to interpret the $\mathrm{CO} / \mathrm{PdGa}(111)$ and $(-1-1-1)$ systems as follows: On $\mathrm{Pd}_{3}$, the adsorption site is coverage dependent, the 3 -fold hollow site is occupied at low coverage, and the on-top site is occupied if two or three $\mathrm{CO}$ are present on one $\mathrm{Pd}$ trimer. $\mathrm{Pd}_{1}$ allows only for on-top adsorption on the terminating $\mathrm{Pd}$ atom. According to DFT, in all $\mathrm{CO}$ binding conformations the molecular axis is oriented perpendicular to the surface (see insets in Figure 2).

Along with this interpretation, details in the RAIRS results shown in Figure 2 can be explained. The frequency shift from initial to full coverage of the peaks originating from on-top site species amounts to $14 \mathrm{~cm}^{-1}$ for $\mathrm{CO} / \mathrm{Pd}_{1}$ and $21 \mathrm{~cm}^{-1}$ for $\mathrm{CO} /$ $\mathrm{Pd}_{3}$. The full coverage is obtained after exposure to 0.6 and 1.2 $\mathrm{L}$ for $\mathrm{Pd}_{1}$ and $\mathrm{Pd}_{3}$, respectively. On $\mathrm{Pd}_{1}$, the shift is assigned to interactions with the nearest neighbor adsorbates (1-6 CO) separated by $0.69 \mathrm{~nm}$. For $\mathrm{CO}$ adsorbed at the on-top site on $\mathrm{Pd}_{3}$, the main contribution to the shift is attributed to the occupancy increase from two to three $\mathrm{CO}$ per trimer at a mutual distance of $0.31 \mathrm{~nm}$.

Both on-top site peaks exhibit a shoulder at lower wavenumbers, which for $\mathrm{CO} / \mathrm{Pd}_{1}$ shifts along with the main peak, but for $\mathrm{CO} / \mathrm{Pd}_{3}$, remains at a fixed position. These side peaks are assigned to $\mathrm{CO}$ molecules neighboring, for example, a surface vacancy ${ }^{15}$ or a step edge. ${ }^{28}$

The coverage-dependent transition from hollow to on-top site adsorption and the small vibrational shifts due to weak $\mathrm{CO}-\mathrm{CO}$ interactions are in agreement with high resolution electron energy loss spectroscopy (HREELS) of CO adsorbed on PdAg surface alloys of different Pd concentrations. ${ }^{10}$

Additionally, the $\mathrm{CO}$ binding energies shown in Table 1 are comparable to those computed for $\mathrm{PdAg} / \mathrm{Pd}(111)$ surface alloys. ${ }^{11}$ Using the same PBE functionals, Mancera et al. ${ }^{11}$ found on-top site binding energies of 1.37 and $1.29 \mathrm{eV}$ for $\mathrm{CO}$ on single Pd atoms and trimers, respectively. These energies are in very good agreement with our calculations (1.37 and 1.25 $\mathrm{eV})$ for PdGa. On the other hand, the hollow site adsorption energy deviates with $1.97 \mathrm{eV}$ for PdAg from the value of 1.59 $\mathrm{eV}$ found for PdGa. This difference might be a consequence of the reduced $\mathrm{Pd}-\mathrm{Pd}$ distance in the trimer of the surface alloy, and/or of the different ligand effects of $\mathrm{Ag}$ and $\mathrm{Ga}$.

To get a broader insight into the adsorption energy landscape, the binding energies for other possible sites were calculated using DFT. A summary of all tested configurations is given in the Supporting Information. On $\mathrm{Pd}_{1}$, the trimers of $\mathrm{Pd}$ atoms, lying $143 \mathrm{pm}$ below the $\mathrm{Pd}_{1}$ termination, are found less favorable for $\mathrm{CO}$ adsorption $\left(E_{\mathrm{b}}=0.88 \mathrm{eV}\right)$, since the atoms of this third-outermost layer are almost completely coordinated with bulk atoms. Furthermore, $\mathrm{CO}$ adsorbed on the hollow site of the Ga trimers of the second-outermost layer (53 and $56 \mathrm{pm}$ below the surface layer on $\mathrm{Pd}_{3}$ and $\mathrm{Pd}_{1}$, respectively) was 
computed to have a weaker binding energy on both terminations $\left(E_{\mathrm{b}}=0.08 \mathrm{eV}\right.$ for $\mathrm{Pd}_{3}$ and $0.56 \mathrm{eV}$ for $\left.\mathrm{Pd}_{1}\right)$. To investigate the absence of $\mathrm{CO}$ bridge site bonding on $\mathrm{Pd}_{3}$, the binding energies for two $\mathrm{CO}$ molecules in "top-and-bridge" and "top-and-top" configuration on the Pd trimer were computed. For both situations, a local energetic minimum was found, with the "top-and-top" configuration being preferred by $63 \mathrm{meV}$ per $\mathrm{CO}$ molecule. A single molecule located on the trimer in a PdPd bridge site was found unstable in the DFT optimization. The large relative differences in binding energies between the first and the second preferred adsorption sites found by DFT confirms the experimental observation that both surfaces exhibit saturation after adsorption of one and three $\mathrm{CO}$ molecules per surface unit cell, respectively.

Krajci and Hafner conducted a computational study on the structure and adsorption properties of the 3-fold PdGa surface. ${ }^{30}$ Their adsorption energies for $\mathrm{CO}$ on the $\mathrm{Pd}_{1}$ and $\mathrm{Pd}_{3}$ surface are in agreement with our DFT derived values summarized in Table 1, with a shift to lower binding energies, which is consistent with the different parametrization for the $\mathrm{PBE}$ approximation. However, in their study they concluded Ga surface terminations to be energetically more favorable than the Pd ones, which we find to be incompatible with experiment (see Supporting Information). ${ }^{15,19}$

We studied the coverage dependence for $\mathrm{CO} / \mathrm{Pd}_{3}$ in more detail by LT-STM. Images at higher coverage than in Figure 1 reveal four different $\mathrm{CO}$ occupations per Pd-trimer, namely, one, two, three, and no CO molecules per trimer (cf. Figure 3). As already shown in Figure 1, we find that one $\mathrm{CO}$ per $\mathrm{Pd}$ trimer appears as a single protrusion that is centered on the $\mathrm{Pd}_{3}$ trimer. If two $\mathrm{CO}$ molecules are adsorbed on a Pd trimer, the imaged adsorbate has a larger apparent height and an elliptical shape (Figure 3A). The center of the protrusion is found slightly off the $\mathrm{Pd}_{3}$ hollow sites located at the intercepts of the red grid (Figure 3B). Additionally, their long semiaxis is not oriented along the $\langle 1-10\rangle$ directions, but along the lines connecting two of the three $\mathrm{Pd}$ atoms in the trimer. The sketch in Figure $3 \mathrm{C}$ shows the orientation of trimers as determined by LEED-I(V). ${ }^{15}$ Together with the change in IR-absorption frequency, we conclude that adding one more $\mathrm{CO}$ molecule per $\mathrm{Pd}_{3}$ converts the 3-fold hollow site to three on-top sites of which two are occupied. Higher coverage leads to wider, 3-fold symmetric protrusions (Figure 3D), exhibiting the same orientation as the $\mathrm{Pd}_{3}$ trimers, which is consistent with the hypothesis of three $\mathrm{CO}$ molecules in the on-top site configuration on the topmost Pd atoms. Accordingly, STM confirms on a local scale the adsorption site sequence derived from RAIRS and DFT simulations for $\mathrm{CO} / \mathrm{Pd}_{3}$. As seen from the STM topographies for $\mathrm{CO}$ on $\mathrm{Pd}_{3}$ and $\mathrm{Pd}_{1}$, shown in Figures 1 and 3 , CO molecules are found exclusively at the positions of the protrusions on the clean surfaces. As these protrusions coincide with the outermost $\mathrm{Pd}$ atoms, ${ }^{15}$ we find no indication for bonding of $\mathrm{CO}$ to $\mathrm{Ga}$.

The presented data show different expression of the active site separation on the $\mathrm{Pd}_{3}$ and $\mathrm{Pd}_{1}$ terminations. At low coverage, the adsorption sites have the same separation on both surfaces (one $\mathrm{CO}$ per unit cell), but are of a different type, namely, on-top site for $\mathrm{Pd}_{1}$ and hollow site for $\mathrm{Pd}_{3}$. However, the $\mathrm{Pd}_{3}$ surface also has three adjacent $\mathrm{Pd}$ on-top adsorption sites that are populated at high coverage; therefore it can accommodate three times as many $\mathrm{CO}$ than the $\mathrm{Pd}_{1}$ surface. This means that at full $\mathrm{CO}$ coverage the minimum $\mathrm{CO}-\mathrm{CO}$
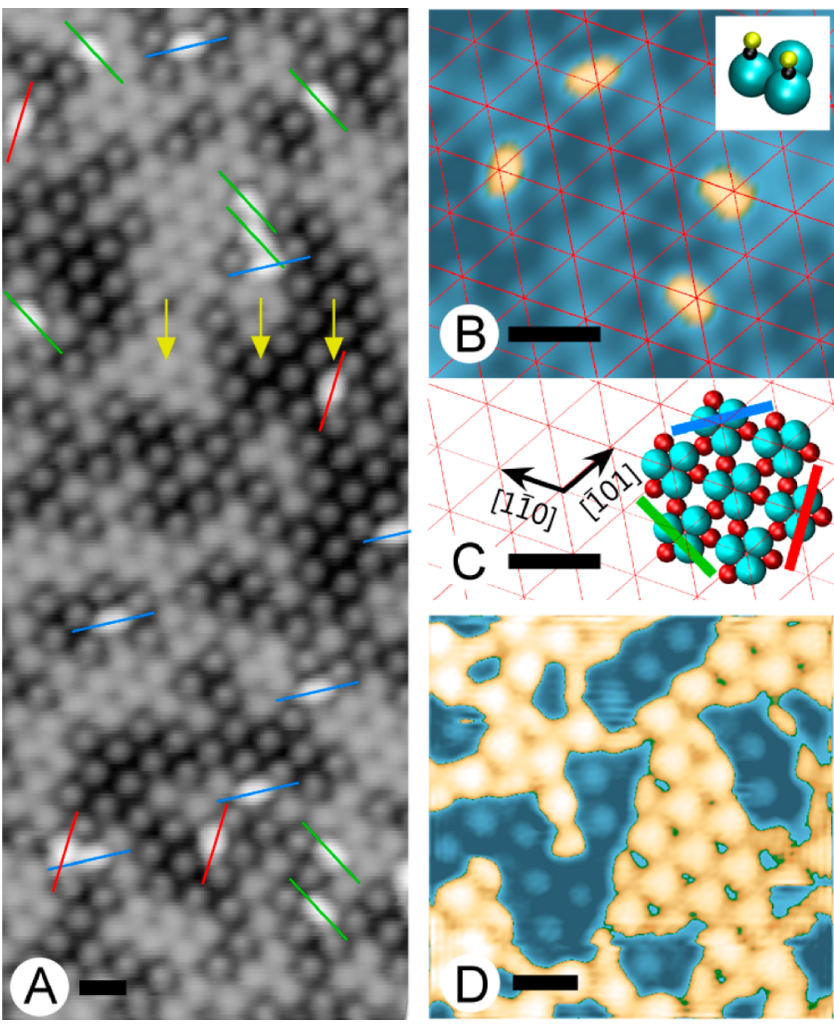

Figure 3. (A) LT-STM image of a partially CO covered PdGa:B(111)$\mathrm{Pd}_{3}$ surface. A $\mathrm{Pd}_{3}$ trimer with no $\mathrm{CO}$ (left arrow) appears in lightgray. A trimer with one $\mathrm{CO}$ (center arrow) appears as a well localized protrusion with lower apparent height, and a trimer with two $\mathrm{CO}$ (right arrow) as elongated white protrusion. The different elongated protrusions appear in three distinct directions indicated by the colored lines. These directions coincide with the "edges" of a Pd trimer (see C). (B) Detail showing that elliptical protrusions are off center with respect to the $\mathrm{Pd}_{3}$ lattice. (C) Surface atomic structure derived from LEED-I(V). ${ }^{15}$ (D) Increased CO exposure leads to protrusions with triangular envelopes, that is, fully occupied trimers. Scale bars correspond to $1.0 \mathrm{~nm}$. In $\mathrm{B}$ and $\mathrm{C}$, a different color-scale was chosen to emphasize the shape of the $\mathrm{CO}$ protrusions (beige; tunneling parameters: (A, B) $0.2 \mathrm{~V}, 0.5 \mathrm{nA}$; (D) $-2.5 \mathrm{~V}, 0.5 \mathrm{nA}$ ).

separation is $0.69 \mathrm{~nm}$ for the $\mathrm{Pd}_{1}$ surface and only $0.31 \mathrm{~nm}$ for the $\mathrm{Pd}_{3}$ surface.

To investigate the chemical behavior of the active sites we characterized the nature of the binding of $\mathrm{CO}$ to the $\mathrm{Pd}$ sites by computing the covalent bond-order index, following the approach by Angyan et al. ${ }^{29}$ in the framework of Bader's topological theory. The method is basis-set independent and takes into account bond ionicities, leading to reduced bond orders for polar covalent bonds. For $\mathrm{CO}$ adsorbed on PdGa, the computed covalent $\mathrm{Pd}-\mathrm{C}$ bond order reveals that the bonding character in the case of one $\mathrm{CO}$ molecule per surface unit cell is remarkably different on the two surfaces, whereas the on-top site bond configuration for high coverage is very similar. In the $\mathrm{Pd}_{1}$ case, our analysis indicates a bond order of 0.83 for the $\mathrm{Pd}-\mathrm{C}$ bond (where a value of 2.89 would correspond to $\mathrm{C}-\mathrm{C}$ triple bond, as in acetylene). In the $\mathrm{Pd}_{3}$ case, when $\mathrm{CO}$ is adsorbed at the hollow site of the trimer, each $\mathrm{Pd}$ atom contributes a bond order of 0.45 , for a total bond order of 1.34. Indeed, the calculated adsorption energy in this configuration is higher compared to $\mathrm{Pd}$ on-top and the IR active $\mathrm{CO}$ vibrational mode has a lower frequency due to the weakened $\mathrm{C}-\mathrm{O}$ bond, which is indicated by a small reduction 
of the $\mathrm{C}-\mathrm{O}$ covalent bond order (cf. Table 1). In the case of three $\mathrm{CO}$ per $\mathrm{Pd}_{3}$, the situation is again similar to $\mathrm{CO}$ on $\mathrm{Pd}_{1}$, which is reflected in binding energy, vibrational mode frequency and a comparable bond-order of 0.82 and 0.83 , respectively.

For both 3-fold PdGa surfaces the site isolation concept is realized for the $\mathrm{CO}$ adsorption. This can be seen in particular by comparing it to $\mathrm{CO}$ adsorption on $\mathrm{Pd}(111)$. In the case of the $\mathrm{Pd}_{1}$ surface, full $\mathrm{CO}$ coverage is obtained at a much lower molecular density of $2.41 \mathrm{CO} / \mathrm{nm}^{2}$ compared to $15.26 \mathrm{CO} /$ $\mathrm{nm}^{2}$ for $\mathrm{Pd}(111)$, with both cases having on-top Pd adsorption. In the case of the $\mathrm{Pd}_{3}$ surface the $\mathrm{CO}$ adsorption proceeds from hollow site adsorption on the Pd trimer to on-top site adsorption with increasing coverage, reaching a maximum $\mathrm{CO}$ density of $7.23 \mathrm{CO} / \mathrm{nm}^{2}$. Although the $\mathrm{CO}$ density on the $\mathrm{Pd}_{3}$ surface is a factor of 2 lower compared to $\mathrm{Pd}(111)$, the nearest $\mathrm{CO}-\mathrm{CO}$ distance is only $10 \%$ different $\left(\mathrm{Pd}_{3}, 0.31 \mathrm{~nm}\right.$; $\operatorname{Pd}(111), 0.275 \mathrm{~nm})$. The reason for the very different molecular saturation densities is the large separation between the trimers on $\mathrm{Pd}_{3}$. While the local Pd trimers on $\mathrm{Pd}_{3}$ do not show bridge site adsorption for $\mathrm{CO}$, this is observed on $\operatorname{Pd}(111)$. Therefore, $\operatorname{Pd}(111)$ exhibits an almost continuous transition from hollow to bridge to on-top site adsorption with increasing coverage. ${ }^{23,24}$

This transition was also reported for Pd ensembles on PdAu and PdAg surface alloys. ${ }^{3,8,10}$ In these alloys, the Pd ensemble size depends on the random local $\mathrm{Pd}$ concentration at the surface, whereas in the PdGa (111) and (-1-1-1) surfaces, size and separation are given by the crystal structure and do not allow for $\mathrm{CO}$ bridge site adsorption. However, the $\mathrm{Pd}$ ensembles found in the partially covalently bound IMC retain their adsorption properties, as adsorption sites, energy, and vibrational modes compare for $\mathrm{CO}$ adsorbed on the IMC and on the surface alloys, for Pd ensembles of equal size.

\section{CONCLUSIONS}

We compared the two 3-fold-symmetric surfaces of the PdGa IMC with regard to active adsorption site separation and ensemble effect. $\mathrm{CO}$ was used as a test molecule, confirming the active site isolation ${ }^{12}$ and revealing differences in the ensemble effect for the two surface structures exhibiting single atoms $\left(\mathrm{Pd}_{1}\right)$ and trimers $\left(\mathrm{Pd}_{3}\right)$. Our combined analysis of STM, RAIRS, and DFT shows that $\mathrm{Pd}_{1}$ provides, independent of coverage, only one on-top site for $\mathrm{CO}$, whereas $\mathrm{Pd}_{3}$ allows for hollow site adsorption at low coverage and on-top site adsorption at high coverage. This leads to a maximum occupancy of one and three $\mathrm{CO}$ molecules per surface unit cell for $\mathrm{Pd}_{1}$ and $\mathrm{Pd}_{3}$, respectively, while no adsorption is taking place on Ga related sites. This observation underlines the hypothesis that the Ga indeed only assumes the role of a spacer atom with regard to the $\mathrm{CO}$ adsorption properties.

Due to the similar electronic structure of these surfaces, the differences in their bonding properties are mainly attributed to the geometric ensemble effect. Furthermore, the comparison with CO adsorption studies on Pd-containing surface alloys supports the argument of a dominating ensemble- over the ligand effect in this IMC. This concept of the ensemble effect in PdGa combined with the active site isolation represents a paradigm for the study of selective catalytic reactions.

\section{ASSOCIATED CONTENT}

\section{S Supporting Information}

Data on STM topography contrast of CO, details on the computations, and a verification of the $\mathrm{PdGa}: \mathrm{B}(111) \mathrm{Pd}_{3}$ and PdGa:B(-1-1-1) $\mathrm{Pd}_{1}$ surface structures with respect to ref 30 . This material is available free of charge via the Internet at http://pubs.acs.org.

\section{AUTHOR INFORMATION}

\section{Corresponding Author}

*E-mail: roland.widmer@empa.ch. Tel.: +41 587654745.

\section{Present Address}

${ }^{\S}$ Drug Discovery and Development, Italian Institute of Technology, Via Morego 30, 16163 Genova, Italy (R.G.).

\section{Notes}

The authors declare no competing financial interest.

\section{ACKNOWLEDGMENTS}

We gratefully acknowledge funding by the Swiss National Science Foundation under Contract 200021-129511 and support by the Swiss National Supercomputing Center (CSCS).

\section{REFERENCES}

(1) Bocklein, S.; Gunther, S.; Wintterlin, J. High-Pressure Scanning Tunneling Microscopy of a Silver Surface During Catalytic Formation of Ethylene Oxide. Angew. Chem., Int. Ed. 2013, 52, 5518-5521.

(2) Herbschleb, C. T.; Bobaru, S. C.; Frenken, J. W. M. HighPressure STM Study of NO Reduction by CO on Pt(100). Catal. Today 2010, 154, 61-67.

(3) Chen, M. S.; Kumar, D.; Yi, C. W.; Goodman, D. W. The Promotional Effect of Gold in Catalysis by Palladium-Gold. Science 2005, 310, 291-293.

(4) Somorjai, G. A. Surface Science and Catalysis. Science 1985, 227, 902-908.

(5) Ertl, G.; Freund, H. J. Catalysis and Surface Science. Phys. Today 1999, 52, 32-38.

(6) Kyriakou, G.; Boucher, M. B.; Jewell, A. D.; Lewis, E. A.; Lawton, T. J.; Baber, A. E.; Tierney, H. L.; Flytzani-Stephanopoulos, M.; Sykes, E. C. H. Isolated Metal Atom Geometries as a Strategy for Selective Heterogeneous Hydrogenations. Science 2012, 335, 1209-1212.

(7) Sachtler, W. M. H. Chemisorption Complexes on Alloy Surfaces. Catal. Rev.: Sci. Eng. 1976, 14, 193-210.

(8) Ruff, M.; Takehiro, N.; Liu, P.; Norskov, J. K.; Behm, R. J. Sizespecific Chemistry on Bimetallic Surfaces: A Combined Experimental and Theoretical Study. ChemPhysChem 2007, 8, 2068-2071.

(9) Gao, F.; Goodman, D. W. Pd-Au Bimetallic Catalysts: Understanding Alloy Effects from Planar Models and (Supported) Nanoparticles. Chem. Soc. Rev. 2012, 41, 8009-8020.

(10) Ma, Y. S.; Diemant, T.; Bansmann, J.; Behm, R. J. The Interaction of $\mathrm{CO}$ with $\mathrm{PdAg} / \mathrm{Pd}(111)$ Surface Alloys: A Case Study of Ensemble Effects on a Bimetallic Surface. Phys. Chem. Chem. Phys. 2011, 13, 10741-10754.

(11) Mancera, L. A.; Behm, R. J.; Gross, A. Structure and Local Reactivity of PdAg/Pd(111) Surface Alloys. Phys. Chem. Chem. Phys. 2013, 15, 1497-1508.

(12) Kovnir, K.; Armbrüster, M.; Teschner, D.; Venkov, T. V.; Szentmiklosi, L.; Jentoft, F. C.; Knop-Gericke, A.; Grin, Y.; Schlögl, R. In Situ Surface Characterization of the Intermetallic Compound PdGa: A Highly Selective Hydrogenation Catalyst. Surf. Sci. 2009, 603, 1784-1792.

(13) Osswald, J.; Kovnir, K.; Armbrüster, M.; Giedigkeit, R.; Jentoft, R. E.; Wild, U.; Grin, Y.; Schlögl, R. Palladium-Gallium Intermetallic Compounds for the Selective Hydrogenation of Acetylene, Part II: Surface Characterization and Catalytic Performance. J. Catal. 2008, 258, 219-227. 
(14) Borodzinski, A.; Bond, G. C. Selective Hydrogenation of Ethyne in Ethene-Rich Streams on Palladium Catalysts, Part 2: Steady-State Kinetics and Effects of Palladium Particle Size, Carbon Monoxide, and Promoters. Catal. Rev.: Sci. Eng. 2008, 50, 379-469.

(15) Prinz, J.; Gaspari, R.; Pignedoli, C. A.; Vogt, J.; Gille, P.; Armbrüster, M.; Brune, H.; Gröning, O.; Passerone, D.; Widmer, R. Isolated Pd Sites on the Intermetallic PdGa(111) and PdGa(-1-1-1) Model Catalyst Surfaces. Angew. Chem., Int. Ed. 2012, 51, 9339-9343.

(16) Norskov, J. K.; Bligaard, T.; Rossmeisl, J.; Christensen, C. H. Towards the Computational Design of Solid Catalysts. Nat. Chem. 2009, 1, 37-46.

(17) Gille, P.; Ziemer, T.; Schmidt, M.; Kovnir, K.; Burkhardt, U.; Armbrüster, M. Growth of Large PdGa Single Crystals from the Melt. Intermetallics 2010, 18, 1663-1668.

(18) Armbrüster, M.; Borrmann, H.; Wedel, M.; Prots, Y.; Giedigkeit, R.; Gille, P. Refinement of the Crystal Structure of Palladium Gallium (1:1), PdGa. Z. Kristallogr.: New Cryst. Struct. 2010, 225, 617-618.

(19) Rosenthal, D.; Widmer, R.; Wagner, R.; Gille, P.; Armbrüster, M.; Grin, Y.; Schlögl, R.; Gröning, O. Surface Investigation of Intermetallic PdGa(-1-1-1). Langmuir 2012, 28, 6848-6856.

(20) VandeVondele, J.; Krack, M.; Mohamed, F.; Parrinello, M.; Chassaing, T.; Hutter, J. QUICKSTEP: Fast and Accurate Density Functional Calculations Using a Mixed Gaussian and Plane Waves Approach. Comput. Phys. Commun. 2005, 167, 103-128.

(21) Perdew, J. P.; Burke, K.; Ernzerhof, M. Generalized Gradient Approximation Made Simple. Phys. Rev. Lett. 1997, 78, 1396-1396.

(22) Pickett, W. E. Pseudopotential Methods in Condensed Matter Applications. Comput. Phys. Rep. 1989, 9, 115-197.

(23) Hoffmann, F. M. Infrared Reflection-Absorption Spectroscopy of Adsorbed Molecules. Surf. Sci. Rep. 1983, 3, 107-192.

(24) Morkel, M.; Rupprechter, G.; Freund, H. J. Ultrahigh Vacuum and High-pressure Coadsorption of $\mathrm{CO}$ and $\mathrm{H}-2$ on $\mathrm{Pd}-(111)$ : A Combined SFG, TDS, and LEED Study. J. Chem. Phys. 2003, 119, $10853-10866$

(25) Tüshaus, M.; Berndt, W.; Conrad, H.; Bradshaw, A. M.; Persson, B. Understanding the Structure of High Coverage CO Adlayers. Appl. Phys. A 1990, 51, 91-98.

(26) Yudanov, I. V.; Sahnoun, R.; Neyman, K. M.; Rosch, N.; Hoffmann, J.; Schauermann, S.; Johanek, V.; Unterhalt, H.; Rupprechter, G.; Libuda, J.; et al. CO Adsorption on Pd Nanoparticles: Density Functional and Vibrational Spectroscopy Studies. J. Phys. Chem. B 2003, 107, 255-264.

(27) Hammer, B.; Hansen, L. B.; Norskov, J. K. Improved Adsorption Energetics within Density-Functional Theory Using Revised Perdew-Burke-Ernzerhof Functionals. Phys. Rev. B 1999, 59, $7413-7421$.

(28) Armbrüster, M.; Behrens, M.; Cinquini, F.; Föttinger, K.; Grin, Y.; Haghofer, A.; Klötzer, B.; Knop-Gericke, A.; Lorenz, H.; Ota, A.; et al. How to Control the Selectivity of Palladium-based Catalysts in Hydrogenation Reactions: The Role of Subsurface Chemistry. ChemCatChem 2012, 4, 1048-1063.

(29) Angyan, J.; Loos, M.; Mayer, I. Covalent Bond Orders and Atomic Valence Indexes in the Topological Theory of Atoms in Molecules. J. Phys. Chem. 1994, 98, 5244-5248.

(30) Krajci, M.; Hafner, J. Structure and Chemical Reactivity of the Polar Three-Fold Surfaces of GaPd: A Density-functional Study. J. Chem. Phys. 2013, 138, 124703. 